\title{
MIXED CONVECTION ON MHD FLOW WITH THERMAL RADIATION, CHEMICAL REACTION AND VISCOUS DISSIPATION EMBEDDED IN A POROUS MEDIUM
}

\author{
B. ZIGTA \\ Debreberhan University, College of Natural and Computational Science \\ Department of Mathematics \\ P.O. Box 445, ETHIOPIA \\ E-mail:tzigta@yahoo.com
}

\begin{abstract}
In this paper, a theoretical analysis has been made to study the effect of mixed convection MHD oscillatory Couette flow in a vertical parallel channel walls embedded in a porous medium in the presence of thermal radiation, chemical reaction and viscous dissipation. The channel walls are subjected to a constant suction velocity and free stream velocity is oscillating with time. The channel walls are embedded vertically in a porous medium. A magnetic field of uniform strength is applied normal to the vertical channel walls. The nonlinear and coupled partial differential equations are solved using multi parameter perturbation techniques. The effects of physical parameters, viz., the radiation absorption parameter, Prandtl number, Eckert number, dynamic viscosity, kinematic viscosity, permeability of porous medium, suction velocity, Schmidt number and chemical reaction parameter on flow variables viz., temperature, concentration and velocity profile have been studied. MATLAB code is used to analyze theoretical facts. The important results show that an increment in the radiation absorption parameter and permeability of porous medium results in an increment of the temperature profile. Moreover, an increment in the Prandtl number, Eckert number and dynamic viscosity results in a decrement of the temperature profile. An increment in suction velocity results in a decrement of the velocity profile. An increment in the Schmidt number, chemical reaction parameter and kinematic viscosity results in a decrement of the concentration profile.
\end{abstract}

Key words: mixed convection, suction velocity, thermal radiation, chemical reaction and viscous dissipation.

\section{Introduction}

An unsteady free and forced convection MHD flow past a vertical channel wall embedded in a porous medium has been studied widely because of its applications in aeronautics, aerodynamics, petroleum, chemical industry, thermal insulation, cooling of nuclear reactors. The combined free and forced convection flow of water from a vertical plate with variable temperature has been studied in [1]. The combined free and forced convection in a porous medium has been investigated in [2]. The effects of MHD unsteady free convection flow past an infinite vertical plate with constant suction and heat sink have been analyzed in [3]. The radiation effects on mixed convection along a vertical plate with a uniform surface temperature using the Rosseland flux model are investigated in [4]. The effect of MHD on free convection oscillatory Couette flow when the temperature and concentration oscillate with time in the presence of the thermal radiation and chemical reaction has been studied in [5]. The effect of thermal radiation, chemical reaction and viscous dissipation on MHD flow has been studied in [6]. The steady hydromagnetic mixed convection flow in a vertical channel with symmetric and asymmetric wall heating conditions in the presence or absence of heat generation or absorption has been studied in [7]. The combined effects of natural convection and a uniform transverse magnetic field when the magnetic field is fixed relative to the plate or fluid have been discussed in [8]. The effects of thermal diffusion, magnetic field and viscous dissipation on unsteady mixed convection flow past a porous plate with chemical reaction have been investigated in [9]. The effects of thermal radiation on mixed convection flow of an optically dense viscous fluid along a vertical porous plate have 
been studied in [10]. The influence of thermal radiation on the laminar natural and forced convection boundary layer flow of a non-absorbing fluid with variable thermo-physical properties flowing around a heat emitting surface and the solutions for the small and large values of thermal radiation parameter have been investigated in [11]. The effect of radiation on mixed convection from a vertical plate in a saturated porous medium has been studied in [12]. The effect of temperature-dependent viscosity on the mixed convection flow from vertical plate has been investigated in [13].

The study of mixed convective mass and heat transfer on a stretching permeable surface has applications in many engineering processes with industrial applications, viz., polymer extrusion, drawing of copper wires, artificial fibers, paper production, hot rolling, wire drawing, glass fiber, metal extrusion, metal spinning and continuous stretching of plastic films. Heat and mass transfer on a stretching sheet with suction or blowing have been examined in [14]. Exact analytical solutions for the flow and heat transfer near the stagnation point on a stretching/shrinking sheet in a Jeffrey fluid were studied in [15]. Heat transfer in a viscoelastic boundary layer flow over a stretching sheet with viscous dissipation and non uniform heat source is derived in [16]. Analytic solutions of unsteady boundary flow and heat transfer on a permeable stretching sheet with non-uniform heat source/sink have been investigated in [17].

The effect of thermal radiation, chemical reaction and viscous dissipation on free convection MHD flow has been studied in [6]. In this study the combined effect of free and forced convection and permeability of porous medium were not considered.

The main objective of this paper is to study the combined effect of free and forced convection MHD oscillatory flow embedded in a porous medium with periodic temperature and concentration in the presence of thermal radiation, chemical reaction and viscous dissipation.

\section{Mathematical analysis}

Consider two dimensional mixed convection Couette flows of unsteady, incompressible, viscous, electrical conducting, Newtonian, chemical reacting and thermal radiating fluid. The fluid flow is bounded by two infinite vertical channel walls separated by a distance $h$ embedded in a porous medium. Furthermore, the fluid is considered to be a gray in color, to have radiation absorbing emitting nature, but it is a non-scattering medium in the optically thick limit. The Rosseland approximation is used to describe the radiative heat flux in the energy equation. It is also assumed that the radiation heat flux in the $x^{\prime}$ direction is negligible as compared to that in $y^{\prime}$ direction.

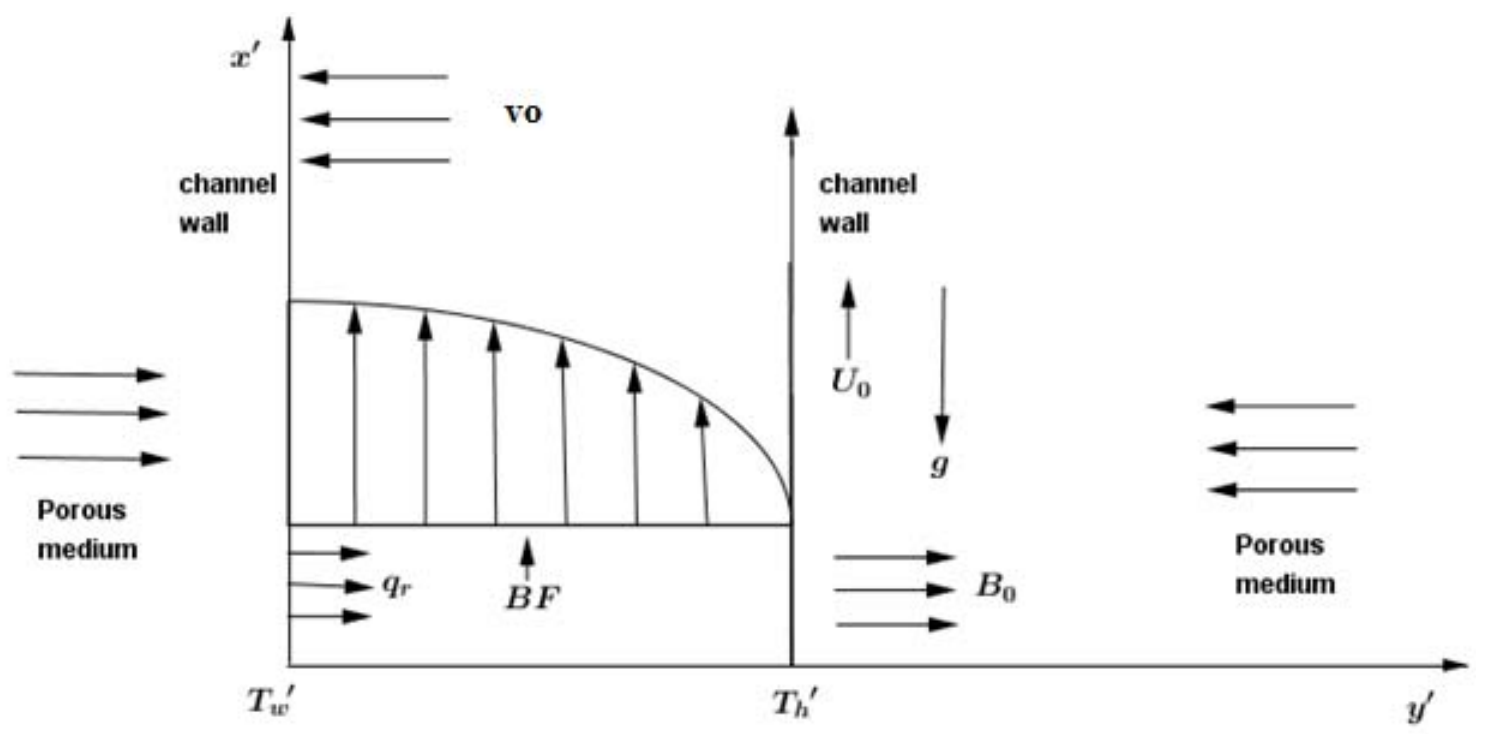

Fig.1. Flow configuration and coordinate system of the model. 
The flow configuration and coordinate system of the model are shown in Fig.1. The $x$ ' axis is taken along the infinite vertical channel wall and the $y^{\prime}$ axis is taken normal to the channel wall. The vertical moving channel wall is located at $y^{\prime}=0$ along the $x^{\prime}$ axis where the temperature is $T_{w}^{\prime}$ and the concentration is $C_{w}^{\prime}$. The other stationary channel wall is located at $y^{\prime}=h$ where the temperature is $T_{h}^{\prime}$ and the concentration is $C_{h}^{\prime}$.

Initially, at $t^{\prime}=0$, the stationary channel wall and the fluid are at the same temperature $T_{h}^{\prime}$ and concentration level of the fluid $C_{h}^{\prime}$ is the same at all points. At a later time $t^{\prime}>0$ the temperature of the moving wall and concentration of the fluid do raise to $T_{w}^{\prime}$ and $C_{w}^{\prime}$, respectively, and are maintained constant thereafter.

Free stream velocity oscillates with time and has the form

$$
U^{\prime}\left(t^{\prime}\right)=U_{o}\left(1+\varepsilon e^{i \omega^{\prime} t^{\prime}}\right)
$$

In Eq.(2.1), $U_{0}$ is the mean constant free stream velocity, $\omega^{\prime}$ is the frequency and $t^{\prime}$ is the time. To derive the governing equations of the model the following assumptions are made:

(1) All fluid properties are constant except the influence of the density variation with temperature and concentration in the body force term.

(2) The Eckert number and magnetic Reynolds numbers are small so that the induced magnetic field of the fluid is negligible.

(3) The external electric field is zero and the electric field due to the polarization of charges is negligible.

(4) Viscosity is also considered with the constant permeability of a porous medium.

(5) There exists a homogeneous chemical reaction of first order with constant rate between diffusing concentration and the fluid in the moving plate.

(6) A uniform magnetic field is applied in the direction perpendicular to the channel walls.

(7) The channel wall is subjected to a constant suction velocity.

(8) All the physical variables are independent of $x^{\prime}$.

Based on the model assumptions and following Sahoo et al. [3] and Soundalgekar (1974), the governing equations of mixed convective flow and heat transfer take the following form:

The continuity equation of the problem has the form

$$
\frac{\partial v^{\prime}}{\partial y^{\prime}}=0
$$

The momentum equation of the problem has the form

$$
\frac{\partial u^{\prime}}{\partial t^{\prime}}+v \frac{\partial u^{\prime}}{\partial y^{\prime}}=\frac{\partial U^{\prime}}{\partial t^{\prime}}+v\left(\frac{\partial^{2} u^{\prime}}{\partial y^{\prime 2}}\right)+g \beta\left(T^{\prime}-T_{h}^{\prime}\right)+g \beta_{c}\left(C^{\prime}-C_{h}^{\prime}\right)-\left(\frac{J X B}{\rho}\right)-\frac{v u^{\prime}}{k^{\prime}} .
$$

In Eq.(2.3), the vector cross product $J x B$ represents the Lorentz force. This term is a body force corresponding to magneto hydrodynamics flow. The total magnetic field is represented by $B$. The density of the current is represented by $J$ and $k^{\prime}$ denotes the permeability of the porous medium. The minus sign in the sixth term of the right hand side indicates that the fluid flows from higher to lower potential. 
As mentioned by Hayat et al. (2008), the expression for the Lorentz force reduces and takes the form as

$$
J x B=-\sigma B^{2} u^{\prime} .
$$

In view of the result (2.4), Eq. (2.3) reduces to

$$
\frac{\partial u^{\prime}}{\partial t^{\prime}}+v \frac{\partial u^{\prime}}{\partial y^{\prime}}=\frac{\partial U^{\prime}}{\partial t^{\prime}}+v \frac{\partial^{2} u^{\prime}}{\partial y^{\prime 2}}+g \beta\left(T^{\prime}-T_{h}^{\prime}\right)+g \beta_{c}\left(C^{\prime}-C_{h}^{\prime}\right)-\frac{\sigma B^{2}\left(u^{\prime}-U^{\prime}\right)}{\rho}-\frac{v u^{\prime}}{k^{\prime}} .
$$

The energy equation of the model can be expressed as

$$
\frac{\partial T^{\prime}}{\partial t^{\prime}}+v \frac{\partial T^{\prime}}{\partial y^{\prime}}=\alpha\left(\frac{\partial^{2} T^{\prime}}{\partial y^{\prime 2}}\right)-\left(\frac{1}{\rho C_{p}}\right)\left(\frac{\partial q_{r}}{\partial y^{\prime}}\right)+\left(\frac{\mu}{\rho C_{p}}\right)\left(\frac{\partial u^{\prime}}{\partial y^{\prime}}\right)^{2} .
$$

The concentration equation of the model can be expressed as

$$
\frac{\partial C^{\prime}}{\partial t^{\prime}}+v \frac{\partial C^{\prime}}{\partial y^{\prime}}=D\left(\frac{\partial^{2} C^{\prime}}{\partial y^{\prime 2}}\right)-K_{r}\left(C^{\prime}-C_{h}^{\prime}\right)
$$

Equations (2.2), (2.5) - (2.7) govern the present model. Here $u^{\prime}$ and $v^{\prime}$ denote the velocity component in the $x^{\prime}$ and $y^{\prime}$ direction, respectively, $\alpha$ - thermal diffusivity, $\rho$ - the density of the fluid, $g-$ the acceleration due to gravity, $\beta$ - the thermal expansion coefficient, $\beta_{c}$ - the concentration expansion coefficient, $T^{\prime}-$ the temperature of the fluid in the boundary layer, $T^{\prime}{ }_{w}-$ the temperature of the moving channel wall, $T_{h}^{\prime}$ - the temperature of the stationary channel wall, $C_{p}$ - the specific heat capacity at constant pressure, $\mu$ - the dynamic viscosity, $\sigma$ - electric conductivity, $q_{r}$ - the local radiative heat flux, $B$ - the magnetic induction, $C^{\prime}-$ the concentration of the fluid in the boundary layer, $C_{w}^{\prime}-$ the concentration of the moving channel wall, $C_{h}^{\prime}$-the concentration of the stationary channel wall, $D-$ the molecular diffusivity, $K_{r}$ - the chemical reaction parameter.

Since the free stream velocity, temperature and concentration fluctuate with time the boundary conditions of the model have the form

$$
y^{\prime}=0, \quad v^{\prime}=-v_{0}, \quad u^{\prime}=U_{o}\left(1+\varepsilon e^{i \omega^{\prime} t^{\prime}}\right)
$$

In Eq.(2.8), $v_{0}$ denotes suction velocity where $v_{0}>0$.

$$
\begin{aligned}
& T^{\prime}=T_{w}^{\prime}+\varepsilon\left(T_{w}^{\prime}-T_{h}^{\prime}\right) e^{i \omega^{\prime} t^{\prime}}, \\
& C^{\prime}=C_{w}^{\prime}+\varepsilon\left(C_{w}^{\prime}-C_{h}^{\prime}\right) e^{i \omega^{\prime} t^{\prime}}, \\
& y^{\prime}=b, \quad u^{\prime}=0, \quad T^{\prime}=T_{h}^{\prime}, \quad C^{\prime}=C_{h}^{\prime} .
\end{aligned}
$$

The Rosseland approximation for radiative heat flux is given by 


$$
q_{r}=\left(\frac{-4 \sigma}{3 k_{s}}\right)\left(\frac{\partial T^{14}}{\partial y^{\prime}}\right)
$$

In Eq.(2.12), the parameters $\sigma$ and $k_{s}$ represent the Stefan Boltzmann constant and the Rosseland mean absorption coefficient, respectively.

Taking the Taylor series expansion of $T^{14}$ and neglecting terms with higher powers, we have

$$
T^{\prime 4} \cong 4 T_{h}^{\prime 3} T^{\prime}-3 T_{h}^{\prime 4}
$$

In view of Eqs (2.12) and (2.13), Eq. (2.6) reduces to

$$
\frac{\partial T^{\prime}}{\partial t^{\prime}}+v \frac{\partial T^{\prime}}{\partial y^{\prime}}=\left(\frac{1}{\rho C_{p}}\right)\left[\kappa+\left(\frac{16 \sigma T_{h}^{\prime 3}}{3 k_{s}}\right)\right]\left(\frac{\partial^{2} T^{\prime}}{\partial y^{\prime 2}}\right)+\left(\frac{Q_{0}}{\rho C_{p}}\right)\left(T^{\prime}-T_{h}^{\prime}\right)+\left(\frac{\mu}{\rho C_{p}}\right)\left(\frac{\partial u^{\prime}}{\partial y^{\prime}}\right)^{2} .
$$

In Eq.(2.14), the third term denotes viscous dissipation.

\section{Non dimensionalization of the model} quantities

The dimensionless form of the model is found by introducing the following non-dimensional

$$
\begin{aligned}
& y=v_{0} y^{\prime} / v ; \quad R a=4 \sigma T_{h}^{\prime 3} / \kappa k_{s} ; \quad u=u^{\prime} / U_{0} ; \quad U=U^{\prime} / U_{0} ; \quad t=t^{\prime} v_{0}^{2} / 4 v ; \quad \omega=4 \omega^{\prime} v / v_{0}^{2} ; \\
& \mathrm{Gc}=\left[\left[g \beta_{c} v\left(C_{w}^{\prime}-C_{h}^{\prime}\right)\right] / U_{0} v_{0}^{2}\right] ; \quad \operatorname{Gr}=\left[g \beta v\left(T_{w}^{\prime}-T_{h}^{\prime}\right) / U_{0} v_{0}^{2}\right] ; \quad \mathrm{Sc}=v / D ; \quad \operatorname{Pr}=v / \alpha ; \\
& M=\sigma B^{2} v / \rho v_{0}^{2} ; \quad C=\left[\left(C^{\prime}-C_{h}^{\prime}\right) /\left(C_{w}^{\prime}-C_{h}^{\prime}\right)\right] ; \quad \mathrm{Ec}=U_{o}^{2} / C_{p} \Delta T ; \quad \Delta T=T_{w}^{\prime}-T_{h}^{\prime} ; \\
& \theta=\left[\left(T^{\prime}-T_{h}^{\prime}\right) /\left(T_{w}^{\prime}-T_{h}^{\prime}\right)\right] ; \quad k=k^{\prime} U_{0}^{2} / v^{2} ; \quad v=\mu / \rho ; \quad Q_{0}=16 \sigma T_{h}^{\prime 2} / k_{s} .
\end{aligned}
$$

Substituting the non-dimensional quantities in Eqs (2.2), (2.5) - (2.7) the equations is reduced to

$$
\begin{aligned}
& \frac{1}{4} \frac{\partial u}{\partial t}-\frac{\partial u}{\partial y}=\frac{1}{4} \frac{\partial U}{\partial t}+\frac{\partial^{2} u}{\partial y^{2}}+\operatorname{Gr} \theta+\operatorname{Gc} C+M(u-U)-\frac{u U_{0}^{2}}{k v_{0}^{2} v^{2}} \\
& \frac{1}{4} \frac{\partial \theta}{\partial t}-\frac{\partial \theta}{\partial y}=\left(\frac{1}{\operatorname{Pr}}+\frac{3 R a k}{v}\right) \frac{\partial^{2} \theta}{\partial y^{2}}+\frac{Q_{0} \theta v}{\rho C_{p} v_{0}^{2}}+\operatorname{Ec} \theta\left(\frac{\partial u}{\partial y}\right)^{2} \\
& \frac{1}{4} \frac{\partial C}{\partial t}-\frac{\partial C}{\partial y}=\frac{1}{\operatorname{Sc}} \frac{\partial^{2} C}{\partial y^{2}}-\frac{K_{r} C v}{v_{0}^{2}}
\end{aligned}
$$


The non-dimensional boundary conditions Eqs (2.8) - (2.11) are

$$
\begin{aligned}
& y=0, \quad u=1+\varepsilon e^{i t}, \quad \theta=1+\varepsilon e^{i t}, \quad C=1+\varepsilon e^{i t}, \\
& y=1, \quad u=0, \quad \theta=0, \quad C=0 .
\end{aligned}
$$

The systems of Eqs (3.1) - (3.3) together with the boundary conditions (3.4) - (3.5) constitute the non-dimensional form of the model.

\section{Analytical solution to the problem}

To find an analytical solution of the non-dimensional form of the present model we consider Eqs (3.1) - (3.5). When the amplitude of oscillations $(\varepsilon \ll 1)$ is very small we can assume the solutions of flow velocity $u$, temperature field $\theta$ and concentration $C$ near the moving channel wall as

$$
\begin{aligned}
& u(y, t)=u_{0}(y)+\varepsilon u_{l}(y) e^{i t}, \\
& \theta(y, t)=\theta_{0}(y)+\varepsilon \theta_{l}(y) e^{i t}, \\
& C(y, t)=C_{0}(y)+\varepsilon C_{l}(y) e^{i t} .
\end{aligned}
$$

In Eqs (4.1) - (4.3), $u_{0}, \theta_{0}$ and $C_{0}$ represent mean velocity, mean temperature and mean concentration, respectively.

Also, the non-dimensional free stream velocity takes the form

$$
U=1+\varepsilon e^{i t}
$$

Substituting Eqs (4.1) - (4.4) into Eqs (3.1) - (3.3), equating harmonic and non-harmonic terms and neglecting higher orders of $\varepsilon$ the following system of equations is obtained

$$
\begin{aligned}
& u_{0}^{\prime \prime}+u_{0}^{\prime}+\operatorname{Gr} \theta_{0}+\operatorname{Gc} C_{0}+M\left(u_{0}-1\right)-\frac{u_{0} U_{0}^{2}}{k v_{0}^{2} v^{2}}=0, \\
& u_{1}^{\prime \prime}+\left(M-\frac{i}{4}\right) u_{1}+\operatorname{Gr} \theta_{1}+\operatorname{Gc} C_{1}+\frac{i}{4}-M=0, \\
& \left(\frac{1}{\operatorname{Pr}}+\frac{3 R a k}{v}\right) \theta_{0}^{\prime \prime}+\theta_{0}^{\prime}+\operatorname{Ec} \theta_{0}\left(\frac{\partial u_{0}}{\partial y}\right)^{2}=0, \\
& \left(\frac{1}{\operatorname{Pr}}+\frac{3 R a k}{v}\right) \theta_{1}^{\prime \prime}+\theta_{1}^{\prime}+\operatorname{Ec} \theta_{1}\left(\frac{\partial u_{1}}{\partial y}\right)^{2}-\frac{i \theta_{1}}{4}=0, \\
& C_{0}^{\prime \prime}+\operatorname{Sc} C_{0}^{\prime}-\frac{K_{r} \operatorname{Sc} C_{0} v}{v_{0}^{2}}=0, \\
& C_{1}^{\prime \prime}+\operatorname{Sc} C_{1}^{\prime}-\operatorname{Sc} C_{1}\left(\frac{K_{r} v}{v_{0}^{2}}+\frac{i}{4}\right)=0 .
\end{aligned}
$$

Further, the boundary conditions corresponding to Eqs (3.4) - (3.5) are obtained as 


$$
\begin{aligned}
& y=0, \quad u_{0}=1, \quad u_{1}=1, \quad \theta_{0}=1, \quad \theta_{1}=1, \quad C_{0}=1, \quad C_{1}=1, \\
& y=1, \quad u_{0}=0, \quad u_{1}=0, \quad \theta_{0}=0, \quad \theta_{1}=0, \quad C_{0}=0, \quad C_{1}=0 .
\end{aligned}
$$

The variables $u_{0}, u_{1}, \theta_{0}, \theta_{1}, C_{0}$ and $C_{1}$ are still coupled in Eqs (4.5) - (4.10). In the case of incompressible fluids, Ec is always very small and it is assumed that

$$
F(y)=F_{0}(y)+\mathrm{Ec} F_{l}(y)+o\left(\mathrm{Ec}^{2}\right) .
$$

Here $F$ stands for any variable $u_{0}, u_{1}, \theta_{0}, \theta_{l}, C_{0}$ and $C_{l}$. These variables can be expanded in powers of Ec as follows

$$
\begin{aligned}
& u_{0}(y)=u_{00}(y)+\operatorname{Ec} u_{01}(y), \\
& u_{1}(y)=u_{10}(y)+\operatorname{Ec} u_{11}(y), \\
& \theta_{0}(y)=\theta_{00}(y)+\operatorname{Ec} \theta_{01}(y), \\
& \theta_{1}(y)=\theta_{10}(y)+\operatorname{Ec} \theta_{11}(y), \\
& C_{0}(y)=C_{00}(y)+\operatorname{Ec}_{01}(y), \\
& C_{1}(y)=C_{10}(y)+\operatorname{Ec}_{11}(y) .
\end{aligned}
$$

Upon substituting Eqs (4.13) - (4.18) in to Eqs (4.5) - (4.10) and equating terms free from Ec and with coefficients $\mathrm{Ec}$ and neglecting higher orders of $\mathrm{Ec}$ the following equations are obtained

$$
\begin{aligned}
& u_{00}^{\prime \prime}+u_{00}^{\prime}+\operatorname{Gr} \theta_{00}+\mathrm{GcC}_{00}+M u_{00}-\frac{u_{00} U_{0}^{2}}{k v_{0}^{2} v^{2}}=0, \\
& u_{01}^{\prime \prime}+u_{01}^{\prime}+\operatorname{Gr} \theta_{01}+\mathrm{GcC}_{01}+M u_{01}-\frac{u_{01} U_{0}^{2}}{k v_{0}^{2} v^{2}}=0, \\
& u_{10}^{\prime \prime}+u_{10}\left(M-\frac{1}{4}\right)+\operatorname{Gr} \theta_{10}+\mathrm{Gc} C_{10}+\frac{1}{4}-M=0, \\
& u_{11}^{\prime \prime}+u_{11}\left(M-\frac{1}{4}\right)+\operatorname{Gr} \theta_{11}+\operatorname{GcC}_{11}+\frac{1}{4}-M=0, \\
& \left(\frac{1}{\operatorname{Pr}}+\frac{3 R a k}{v}\right) \theta_{00}^{\prime \prime}+\theta_{00}^{\prime}=0,
\end{aligned}
$$




$$
\begin{aligned}
& \left(\frac{1}{\operatorname{Pr}}+\frac{3 R a k}{v}\right) \theta_{01}^{\prime \prime}+\theta_{01}^{\prime}+\theta_{00}\left(\frac{\partial u_{00}}{\partial y}\right)^{2}=0, \\
& \left(\frac{1}{\operatorname{Pr}}+\frac{3 R a k}{v}\right) \theta_{10}^{\prime \prime}+\theta_{10}^{\prime}=0, \\
& \left(\frac{1}{\operatorname{Pr}}+\frac{3 R a k}{v}\right) \theta_{11}^{\prime \prime}+\theta_{11}^{\prime}+\theta_{10}\left(\frac{\partial u_{10}}{\partial y}\right)^{2}=0, \\
& C_{00}^{\prime \prime}+\operatorname{Sc} C_{00}^{\prime}-\frac{k_{r} \operatorname{Sc} C_{00} v}{v_{0}^{2}}=0, \\
& C_{01}^{\prime \prime}+\operatorname{Sc}_{01}^{\prime}-\frac{k_{r} \operatorname{Sc} C_{01} v}{v_{0}^{2}}=0, \\
& C_{10}^{\prime \prime}+\operatorname{Sc}_{10}^{\prime}-\operatorname{Sc}_{10}\left(\frac{k_{r} v}{v_{0}^{2}}+\frac{i}{4}\right)=0, \\
& C_{11}^{\prime \prime}+\operatorname{Sc}_{11}^{\prime}-\operatorname{Sc} C_{11}\left(\frac{k_{r} v}{v_{0}^{2}}+\frac{i}{4}\right)=0 .
\end{aligned}
$$

Equations (4.19) - (4.30) are subjected to the boundary conditions

$$
\begin{array}{ll}
y=0, & u_{00}=u_{10}=\theta_{00}=\theta_{10}=C_{00}=C_{10}=1, \quad u_{01}=u_{11}=\theta_{01}=\theta_{11}=C_{01}=C_{11}=0, \\
y=1, & u_{00}=u_{01}=u_{10}=u_{11}=\theta_{00}=\theta_{01}=\theta_{10}=\theta_{11}=C_{00}=C_{01}=C_{10}=C_{11}=0 .
\end{array}
$$

Solving Eqs (4.18) - (4.29) together with the boundary conditions (4.31) - (4.32), the analytical solutions are obtained

$$
\begin{aligned}
& u_{00}(y)=c_{1} e^{\lambda y}+c_{2} e^{-\lambda y}+\frac{\mathrm{Gr} \theta_{00}+\mathrm{Gc}_{00}}{\lambda^{2}}, \\
& u_{01}(y)=c_{1}^{\prime} e^{\lambda y}+c_{2} e^{-\lambda y}+\frac{\mathrm{Gr} \theta_{01}+\mathrm{Gc} C_{01}}{\lambda^{2}}=0, \\
& u_{10}(y)=d_{1} e^{\alpha y}+d_{2} e^{-\alpha y}+\frac{\operatorname{Gr} \theta_{10}+\mathrm{Gc}_{10}+\left(\frac{1}{4}-M\right)}{\alpha^{2}}, \\
& u_{11}(y)=d^{\prime}{ }_{1} e^{\alpha y}+d^{\prime}{ }_{2} e^{-\alpha y}+\frac{\mathrm{Gr}_{11}+\mathrm{Gc}_{11}+\left(\frac{1}{4}-M\right)}{\alpha^{2}}=0, \\
& \theta_{00}(y)=f_{1} e^{\gamma y}+f_{2} e^{-\gamma y}, \\
& \theta_{01}(y)=f^{\prime}{ }_{1} e^{\gamma y}+f^{\prime}{ }_{2} e^{-\gamma y}=0,
\end{aligned}
$$




$$
\begin{aligned}
& \theta_{10}(y)=g_{1} e^{\beta y}+g_{2} e^{-\beta y}, \\
& \theta_{11}(y)=g^{\prime}{ }_{1} e^{\beta y}+g^{\prime}{ }_{2} e^{-\beta y}=0, \\
& C_{00}(y)=h_{1} e^{\xi y}+h_{2} e^{-\xi y}, \\
& C_{01}(y)=h_{1}{ }_{1} e^{\xi y}+h_{2}{ }_{2} e^{-\xi y}=0, \\
& C_{10}(y)=k_{1} e^{\mu y}+k_{2} e^{-\mu y}, \\
& C_{11}(y)=k^{\prime}{ }_{1} e^{\mu y}+k^{\prime}{ }_{2} e^{-\mu y}=0 .
\end{aligned}
$$

In Eqs (4.33) - (4.44) we used the following notations

$$
\lambda=\frac{-1 \pm \sqrt{1-4\left(\frac{M-U_{0}^{2}}{k v_{0}^{2} v^{2}}\right)}}{2} ; \alpha= \pm \sqrt{\frac{1}{4}-M} ; \gamma=\beta=\frac{-\operatorname{Pr} v}{v+3 R a k}, \xi=\frac{-\operatorname{Sc} \pm \sqrt{\operatorname{Sc}\left(\operatorname{Sc}+\frac{4 K_{r} v}{v_{0}^{2}}\right)}}{2}
$$$$
\mu=\frac{-\mathrm{Sc} \pm \sqrt{\mathrm{Sc}\left(\mathrm{Sc}+4\left(\frac{K_{r} v}{v_{0}^{2}}+\frac{i}{4}\right)\right)}}{2} ; \quad c_{1}=\frac{\lambda^{2}-(\mathrm{Gr}+\mathrm{Gc})}{\lambda^{2}\left(1-e^{2 \lambda}\right)} ; \quad c_{2}=\frac{e^{2 \lambda}\left[(\mathrm{Gr}+\mathrm{Gc})-\lambda^{2}\right]}{\lambda^{2}\left(1-e^{2 \lambda}\right)} ;
$$

$$
\begin{aligned}
& d_{1}=\frac{1}{\left(1-e^{2 \alpha}\right)}\left[\left(1-e^{\alpha}\right)+1-\frac{(\mathrm{Gr}+\mathrm{Gc})}{\alpha^{2}}\right] ; \quad d_{2}=\frac{1}{\left(1-e^{2 \alpha}\right)}\left[\frac{(\mathrm{Gr}+\mathrm{Gc}) e^{\alpha}}{\alpha^{2}}-1\right] ; \quad g_{1}=\frac{1}{1-e^{2 \beta}} ; \\
& g_{2}=\frac{e^{2 \beta}}{e^{2 \beta}-1} ; \quad h_{1}=\frac{1}{1-e^{2 \xi}} ; \quad f_{1}=\frac{1}{1-e^{2 \gamma}} ; \quad f_{2}=\frac{e^{2 \gamma}}{e^{2 \gamma}-1} ; \quad h_{2}=\frac{e^{2 \xi}}{e^{2 \xi}-1} ; \\
& k_{1}=\frac{1}{1-e^{2 \mu}} ; \quad k_{2}=\frac{e^{2 \mu}}{e^{2 \mu}-1} .
\end{aligned}
$$

\section{Simulation study of the model}

This study analyzes the combined effect of free and forced convection on MHD oscillatory flow embedded in a porous medium with periodic temperature and concentration in the presence of thermal radiation, chemical reaction and viscous dissipation. The effects of physical parameters, viz., the radiation absorption parameter, Prandtl number, Eckert number, dynamic viscosity, kinematic viscosity, permeability of porous medium, suction velocity, Schmidt number and chemical reaction parameter, on flow variables, viz., temperature, concentration and velocity profile have been studied.

Here only the graphical representations of the temperature profile of the model for different values of the radiation absorption parameter, Eckert number, Prandtl number, permeability of porous medium and dynamic viscosity. Moreover, concentration profiles of the model problem for different values of chemical reaction parameter, kinematic viscosity and Schmidt number and also velocity profile of the model for different values of suction velocity have been presented. 
For the sake of brevity the other parameters such as the Grashof number, i.e., based on temperature difference, the modified Grashof number, i.e., based on concentration difference, magnetic field parameter, skin friction, Sherwood number, Nusselt number and molecular diffusivity have not been discussed.

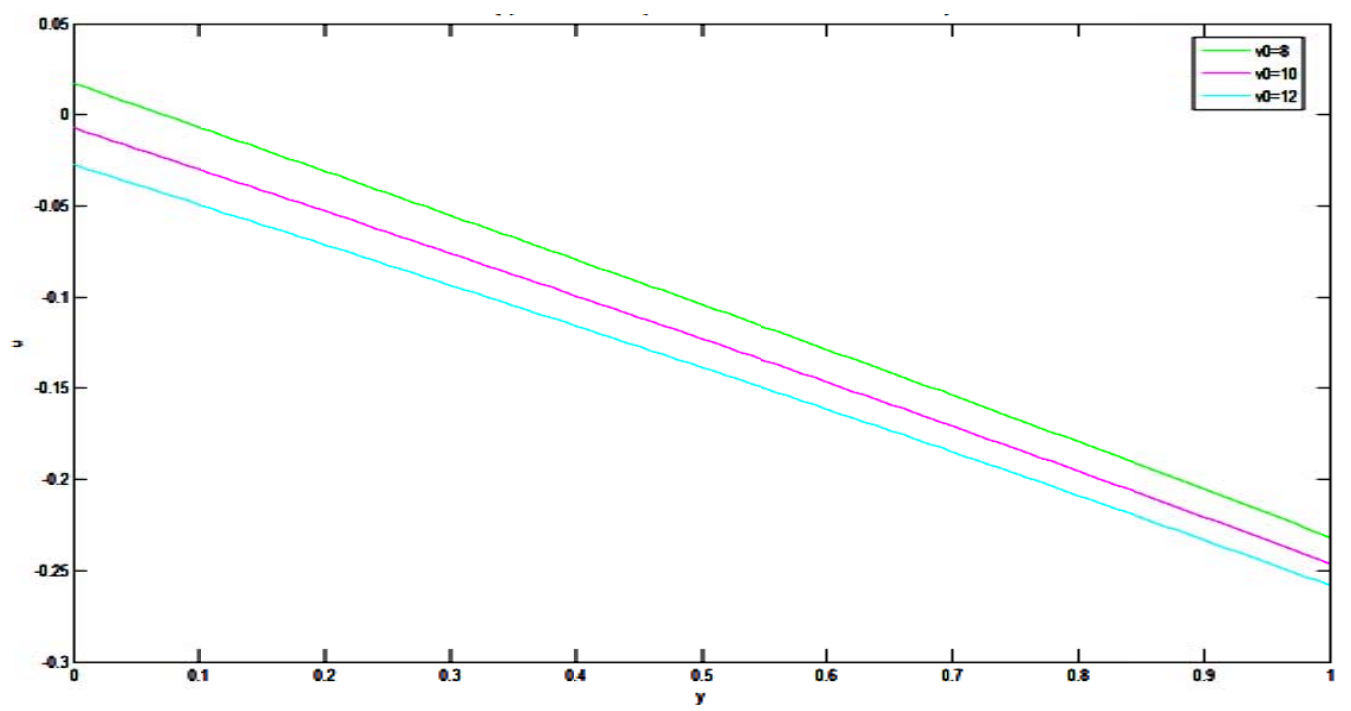

Fig.2. Velocity profile of the model problem for different values of suction velocity.

Figure 2 shows the effect of suction velocity on the velocity profile of the model. The graph of the velocity versus distance between the channel walls for different values of suction velocity has been plotted. From the graph it can be concluded that as suction velocity increases the velocity decreases, i.e., as suction velocity of the fluid flow increases the channel wall is cooled, consequently the viscosity of the flowing fluid increases and there is a gradual decrement of the velocity. Physically, this can be interpreted that the application of suction velocity to a channel wall through openings in the surface stabilizes the hydrodynamic, thermal and concentration boundary layer growth of the fluid flow and thus retards transition from laminar to turbulent flow. Suction velocity reduces the boundary layer thickness and also it helps to develop a laminar velocity profile which is more stable than a profile with no suction.

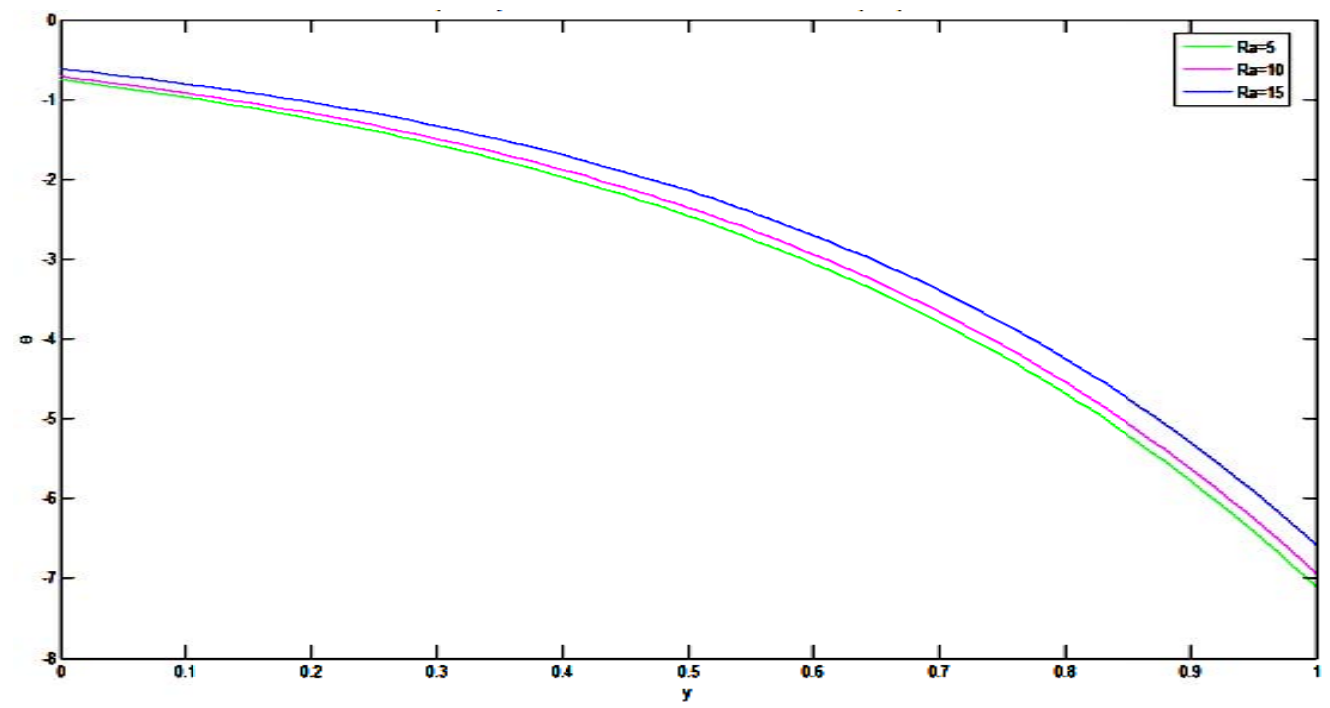

Fig.3. Temperature profile of the model for different values of the radiation absorption parameter. 
In Fig. 3 the influence of the radiation absorption parameter on temperature has been investigated. From the simulated graph it can be observed that as the radiation absorption parameter increases temperature increases. Thermal radiation effects have several practical applications in medical research areas, viz., biomedical engineering, radiotherapy, MRI, CT scan, echocardiography and Doppler ultrasound. Radiation has an important effect on distribution of temperature, i.e., temperature profile increases with the increase of thermal radiation absorption. This results in an increment of thermal boundary layer thickness.

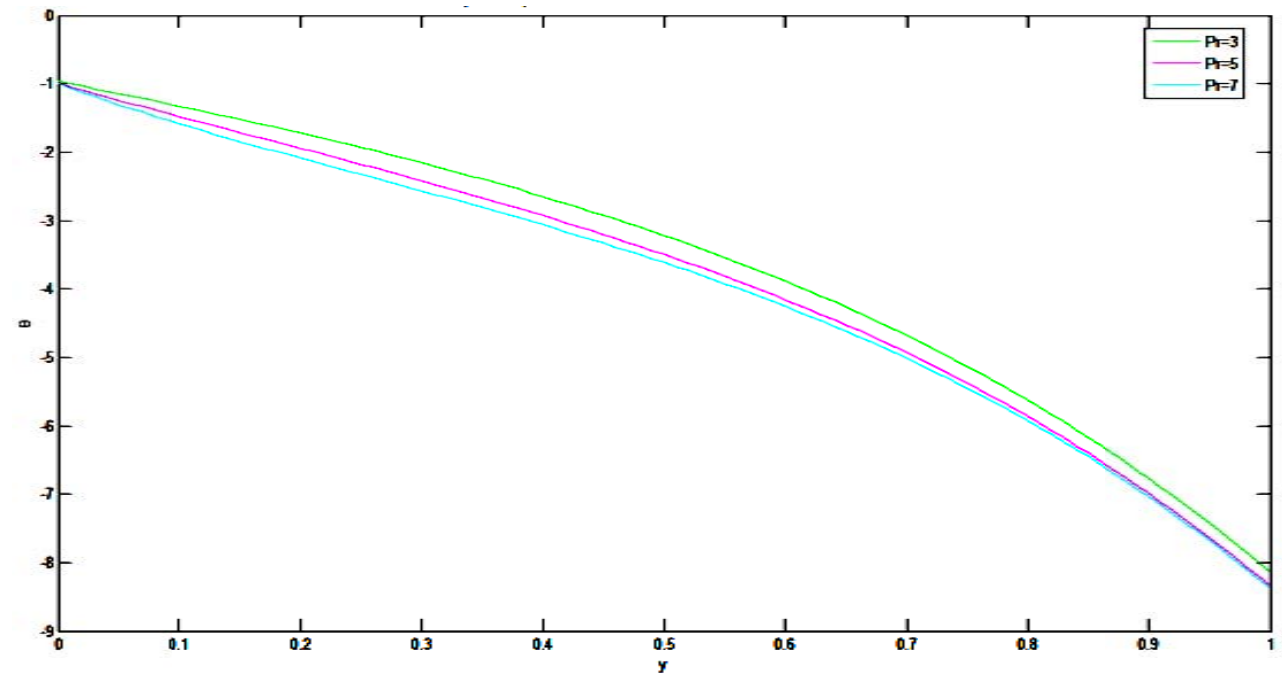

Fig.4. Temperature profile of the model for different values of the Prandtl number.

Figure 4 illustrates the effect of different values of the Prandtl number on the temperature profile. The graph is plotted by taking the distance between the moving and stationary channel wall denoted by $y$ along the horizontal axis and the value of the temperature $\theta$ along the vertical axis. The result shows that for a fixed value of the Prandtl number the temperature $\theta$ starts from a constant value at the moving channel wall, then temperature decreases as $y$ approaches the stationary channel wall. The result of the simulated graph shows that the temperature and Prandtl number are inversely proportional, i.e., as the Prandtl number increases temperature decreases and as the Prandtl number decreases temperature increases. The reason is that larger values of the Prandtl number are equivalent to smaller thermal diffusivity.

Physically, this can be interpreted as follows: thermal energy diffuses more rapidly through materials with high thermal diffusivity than with low thermal diffusivity. Smaller thermal diffusivity implies that thermal energy diffuses slowly which results in a decrement of the thermal boundary layer and also smaller values of the Prandtl number result in larger thermal diffusivity, therefore, heat diffuses away from the heated channel wall. This theoretical fact has been proved successfully by the simulation study.

Figure 5 investigates the temperature profile of the model problem for different values of the Eckert number. From the simulated graph it can be observed that an increment in the Eckert number results in a decrement of the temperature profile. The Eckert number is defined as the ratio of kinetic energy at the wall to the specific enthalpy difference between the wall and fluid. The effect of the Eckert number on temperature can be explained that as the Eckert number increases temperature decreases. The Eckert number physically is a measure of frictional heat in a system. 


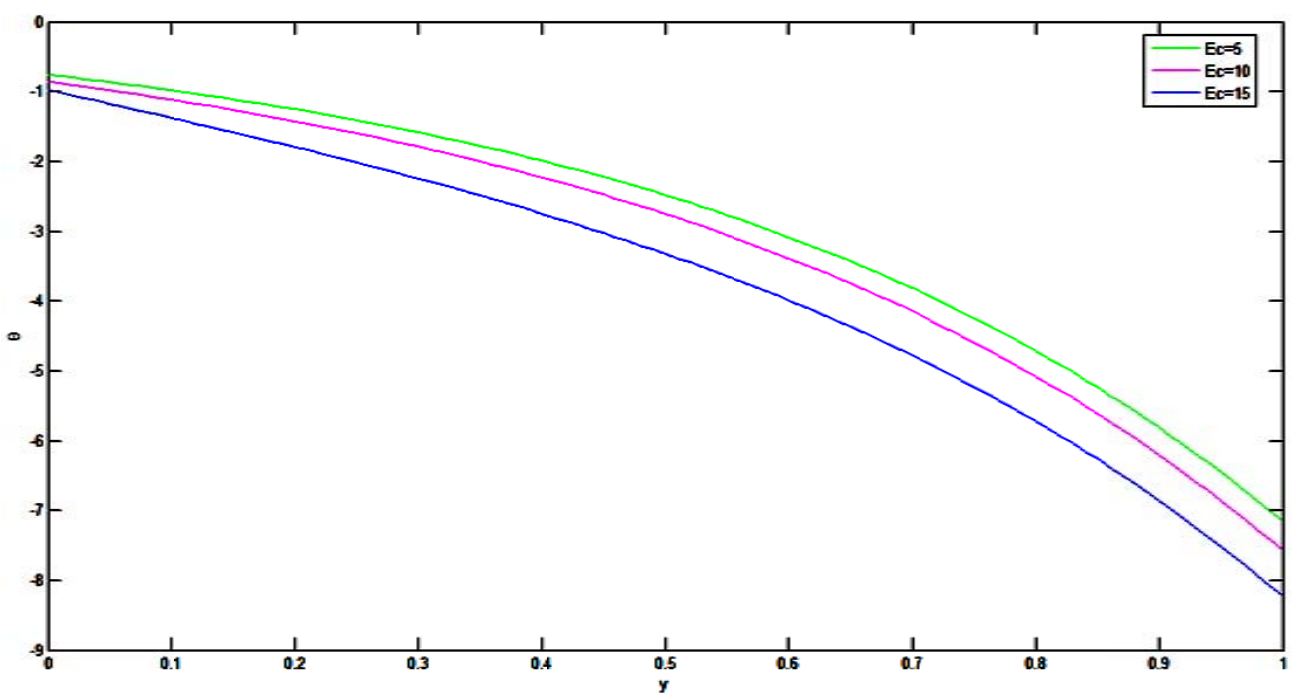

Fig.5. Temperature profile of the model for different values of the Eckert number.

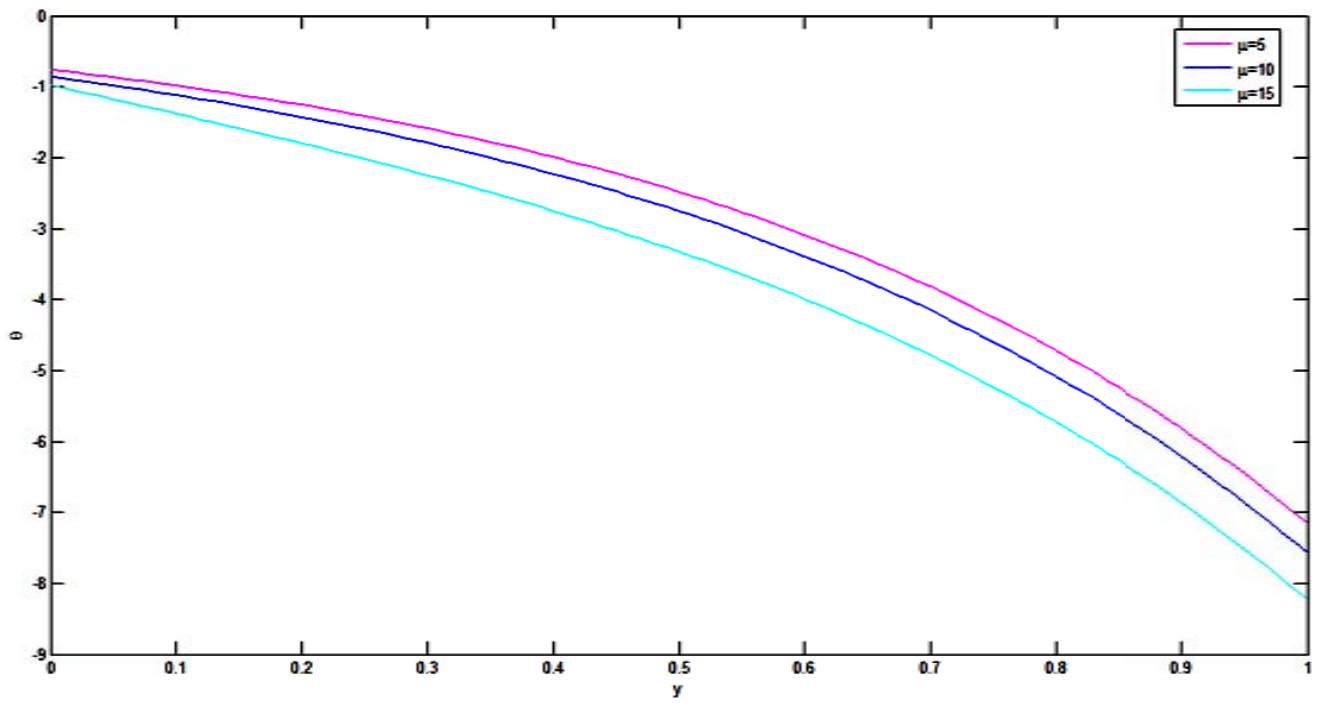

Fig.6. Temperature profile of the model for different values of dynamic viscosity.

Figure 6 shows the temperature profile of the model for different values of dynamic viscosity. It can be observed that an increment in dynamic viscosity results in a decrement of the temperature profile. The Prandtl number is the ratio of the product of dynamic viscosity and specific heat capacity at constant pressure to thermal conductivity. A smaller Prandtl number is equivalent to increasing thermal conductivity and hence this results in increasing temperature. Physically, this can be interpreted as follows: increasing Prandtl number results in increasing dynamic viscosity and hence a decrease in thermal conductivity. This results in decreasing temperature. This fact has been proved by the simulation study. 


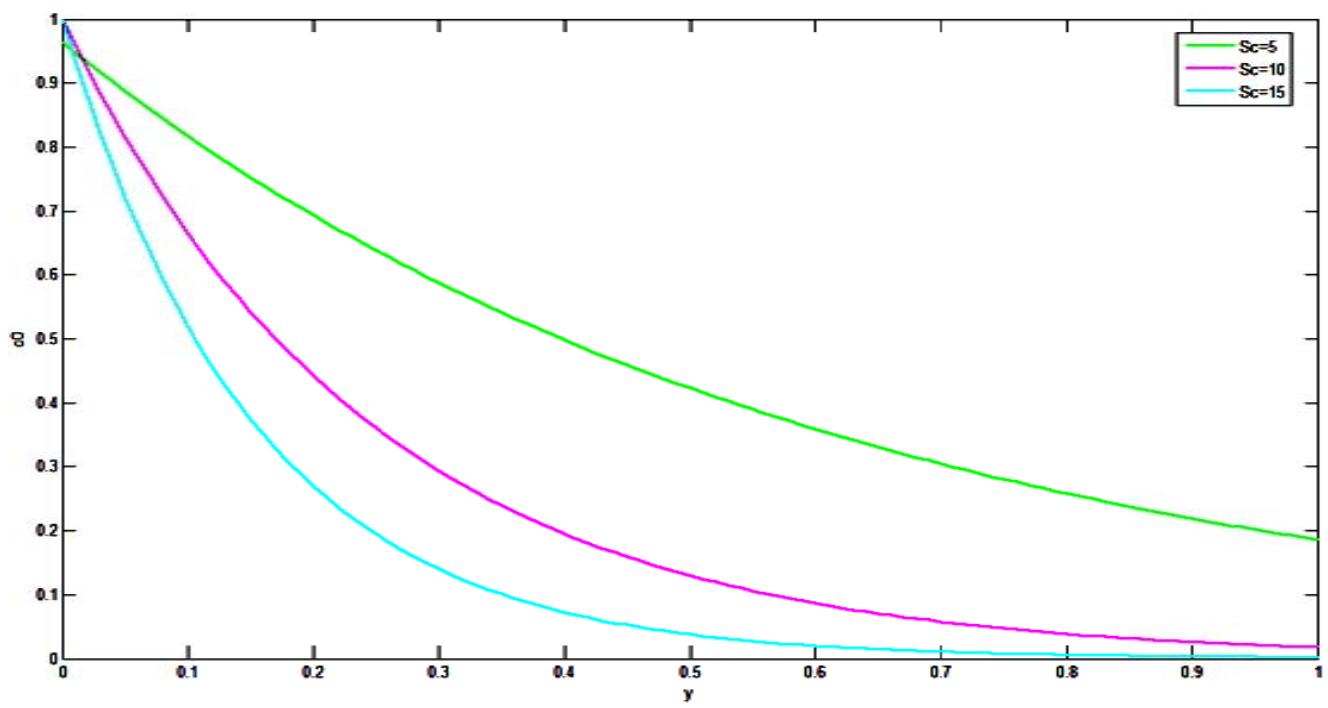

Fig.7. Concentration profile of the model problem for different values of the Schmidt number.

Figure 7 illustrates the influence of the Schmidt number on the concentration profile. The Schmidt number is the ratio of the momentum to molecular diffusivity. Physically, it relates the relative thickness of the hydrodynamic boundary layer and concentration boundary layer. A decrement in the concentration profile results in a decrement of the concentration boundary layer. From the simulation study it can be concluded that as the Schmidt number increases the concentration of the fluid decreases. This causes the concentration buoyancy effects to decrease.

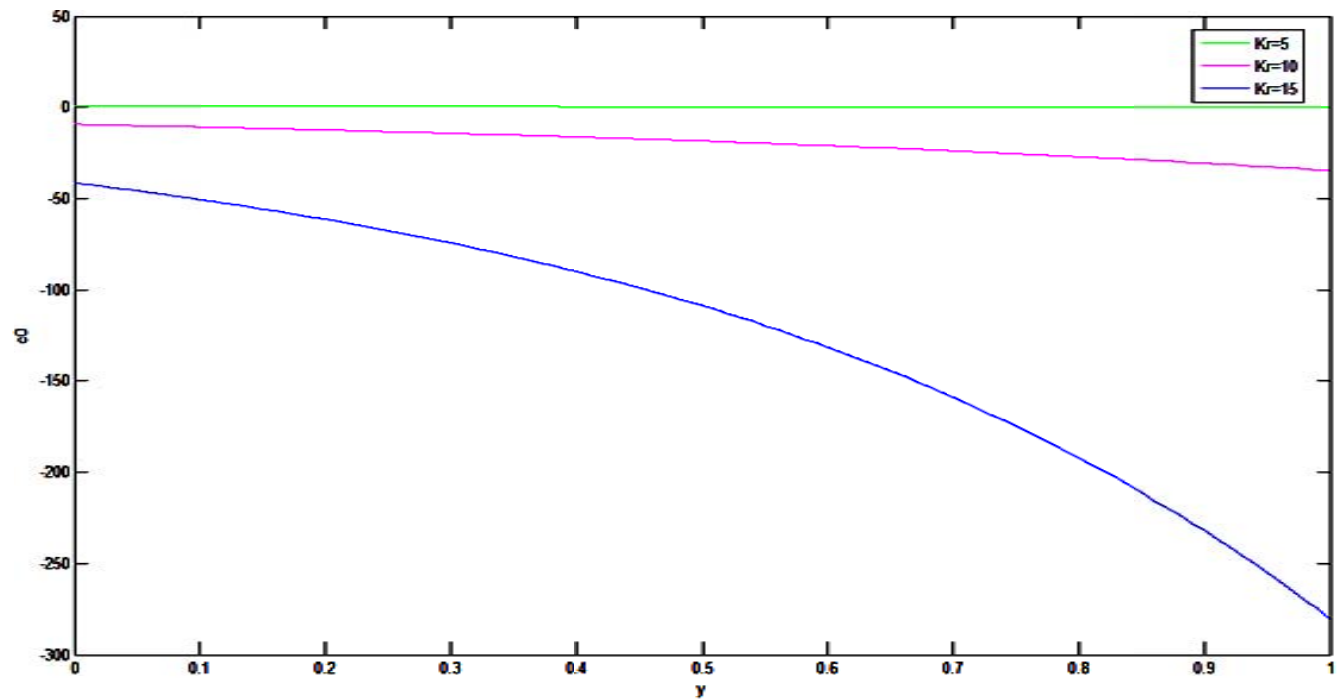

Fig.8. Concentration profile of the model problem for different values of the chemical reaction parameter.

Figure 8 shows the effect of different values of the chemical reaction parameter on the concentration profile. It is observed that an increment in the chemical reaction parameter results in a decrement of the concentration profile. From the figure it can be observed that the concentration distribution decreases at all points of the flow field with an increment in the chemical reaction parameter. This shows that the concentration diffusion with higher values of the chemical reaction parameter has a retarding effect on the concentration distribution in the concentration boundary layer. The physical interpretation of this result indicates that the concentration diffusion is a generative chemical reaction, i.e., the chemical reaction is 
exothermic, thus heat is emitted. On the other hand, concentration diffusion can be a destructive chemical reaction, i.e., the chemical reaction is endothermic and in this case heat is absorbed.

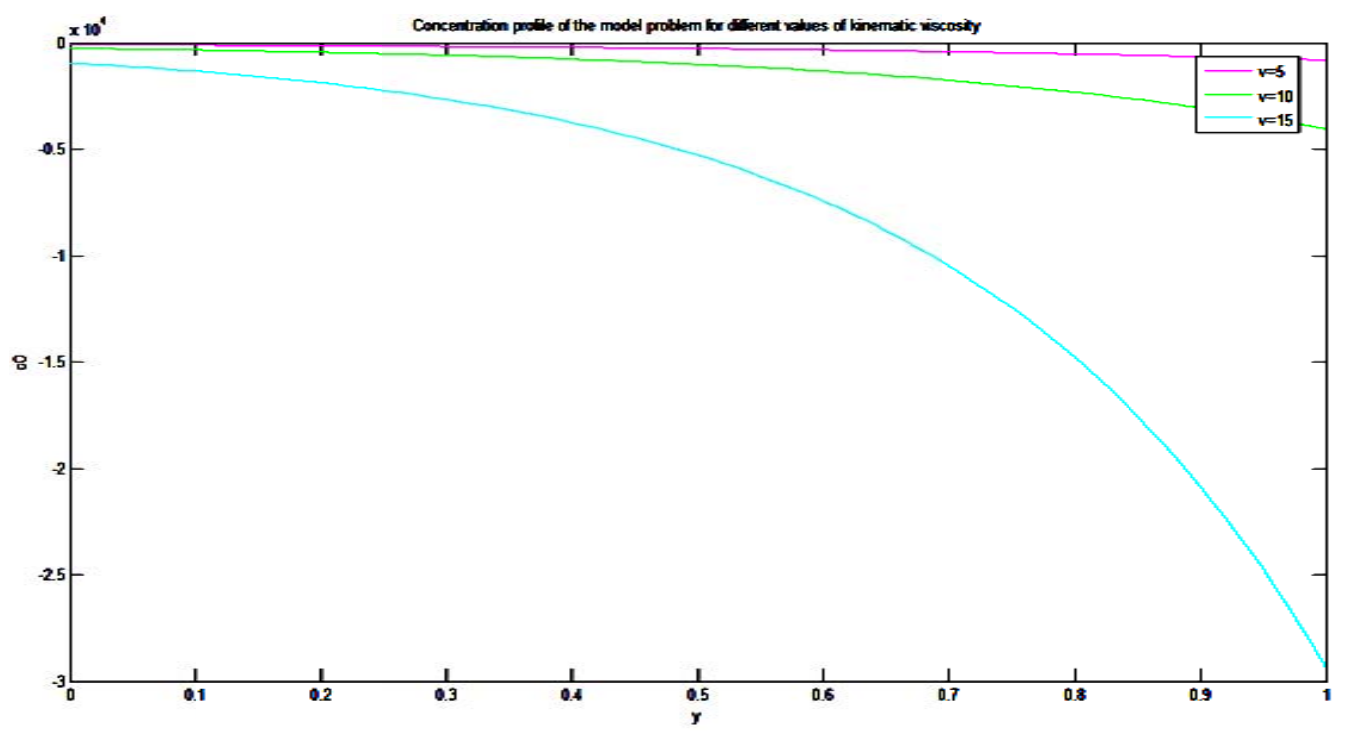

Fig.9. Concentration profile of the model problem for different values of kinematic viscosity.

In Fig.9 the simulated results of the influence of kinematic viscosity on the concentration profile have been studied. Kinematic viscosity is a measure of the resistance to flow of a fluid equal to its absolute viscosity divided by density. From the graph it can be concluded that an increment in kinematic viscosity results in a decrement of concentration. Physically, this can be interpreted as follows: more concentration of a substance in a system results in a greater chance that molecules will collide and speed up the rate of chemical reaction. Furthermore, as a result of an increment in pressure the molecules to have less space to move. A greater density of molecules increases the number of collisions. This results in an increment of concentration. Density and kinematic viscosity are inversely proportional. This fact has been proved by the simulation study.

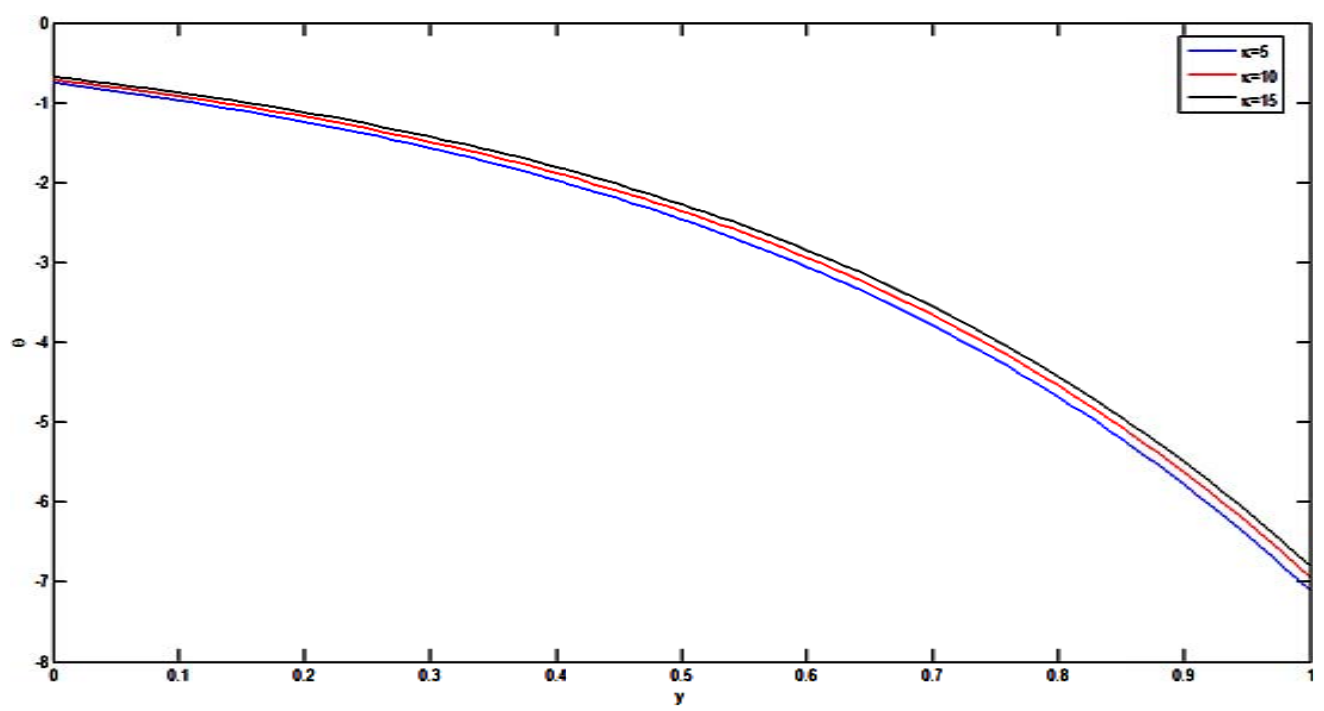

Fig.10. Temperature profile of the model problem for different values of permeability of the porous medium. 
Figure 10 is a graphical representation of the influence of permeability of the porous medium on the temperature profile. From the graph it can be concluded that an increment in permeability of the porous medium results in an increment of temperature. Physically, this means that the resistance dominated by the porous medium reduces as the permeability of the medium increases because of which the velocity increases and consequently the temperature increases.

\section{Conclusion}

This paper studies the effect of mixed convection on a magneto hydrodynamics oscillatory flow embedded in a porous medium with periodic temperature and concentration in the presence of thermal radiation, chemical reaction and viscous dissipation. The effects of various physical parameters, viz., the radiation absorption parameter, Prandtl number, Eckert number, dynamic viscosity, kinematic viscosity, permeability of porous medium, suction velocity, Schmidt number and chemical reaction parameter, on flow variables, viz., temperature, concentration and velocity profile have been studied. Important results of the simulation study are:

(1) An increment in suction velocity results in a decrement of the velocity profile.

(2) An increment in both the radiation absorption parameter and permeability of the porous medium results in an increment of the temperature profile.

(3) An increment in the Prandtl number, Eckert number and dynamic viscosity results in a decrement of the temperature profile.

(4) An increment in the Schmidt number, chemical reaction parameter and kinematic viscosity results in a decrement of the concentration profile.

\section{Application of the model problem}

The model problem can be applied to MHD flow of blood in a permeable capillary as follows:

(1) When suction velocity increases the velocity of blood in the capillary decreases.

(2) When the Prandtl number increases the temperature of the blood in the boundary layer decreases.

(3) The temperature of blood in the capillary increases with an increment of permeability of the porous medium.

(4) As thermal radiation increases, the thermal boundary layer also increases. Minimizing the radiation absorption parameter helps to treat patients with blood cancer and tumor by using thermal therapy.

\section{Nomenclature}

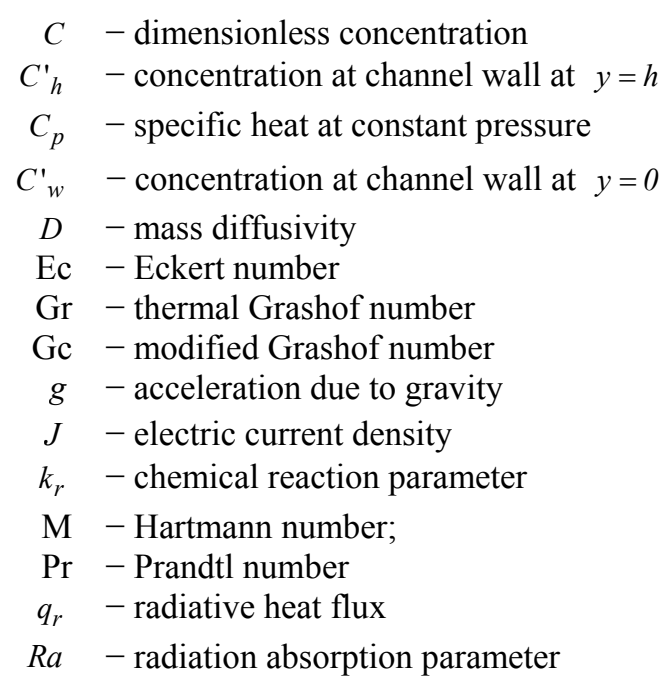




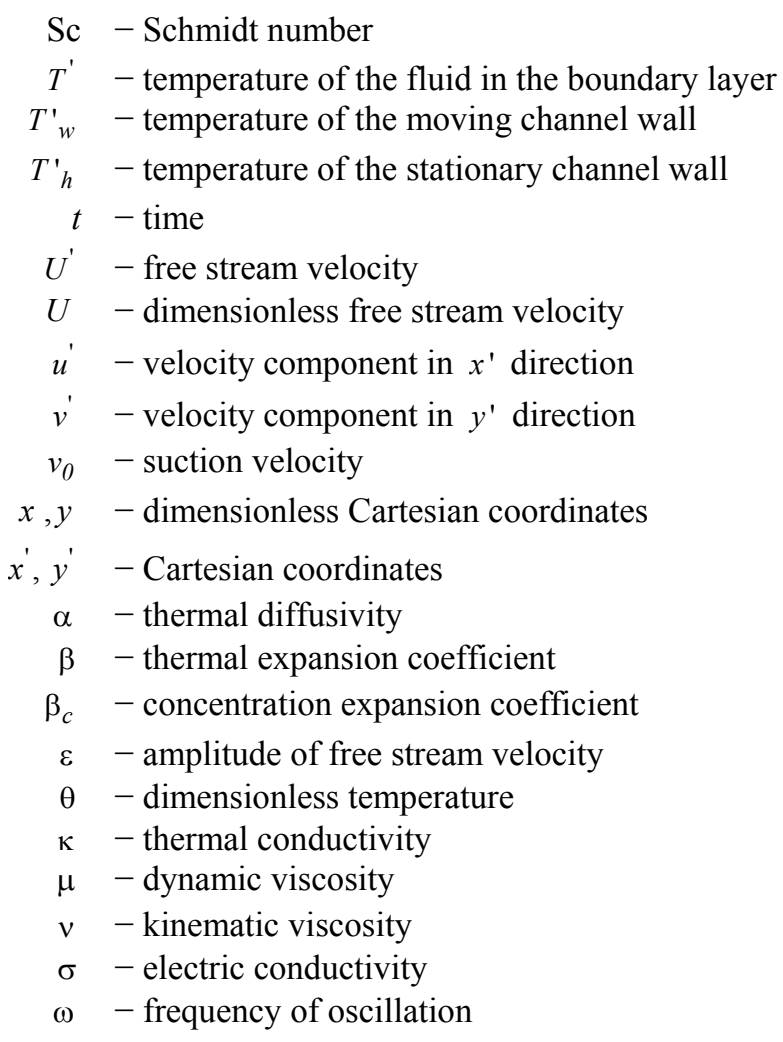

\section{References}

[1] Vighnesam N.V. and Soundalgekar V.M. (1998): Combined free and force convection flow of water at $4^{0} \mathrm{C}$ from a vertical plate with variable temperature. - Int. J. of Engineering and Material Sciences, vol.5, pp.124-126.

[2] Combarnous M.A. and Bia P. (1971): Combined free and forced convection in porous media. - Sot. Petrol. Engng JI vol.11, pp.399-405.

[3] Sahoo P.K., Datta N. and Biswal S. (2003): MHD unsteady free convection flow past an infinite vertical plate with constant suction and heat sink. - IJPAM, vol.34, pp.145-55.

[4] Hossain M.A. and Takhar H.S. (1996): Radiative effects on mixed convection along a vertical plate with uniform surface temperature. - J. Heat and Mass Transfer, vol.31, No.4, pp.243-248.

[5] Zigta B. and Koya P.R. (2017): The effect of MHD on free convection with periodic temperature and concentration in the presence of thermal radiation and chemical reaction. - International Journal of Applied Mechanics and Engineering, vol.22, No.4, pp.1059-1073. DOI: 10.1515/ijame-2017-0068.

[6] Zigta B. (2018): The effect of thermal radiation, chemical reaction and viscous dissipation on MHD flow. International Journal of Applied Mechanics and Engineering, vol.23, No.3, pp.787-801. DOI: 10.2478/ijame2018-0043.

[7] Chamkha A.J. (2002): On laminar hydromagnetic mixed convection flow in a vertical channel with symmetric and asymmetric wall heating conditions. - Int. J. Heat Mass Transfer, vol.45, pp.2509-2525.

[8] Jha B.K. (2001): Natural convection in unsteady MHD Couette flow. Heat and Mass Transfer, vol.37, pp.329-331.

[9] Olanrewaju P.O., Alao F.I. and Adeniyan A. (2013): Effects of thermal diffusion, diffusion thermo, magnetic field and viscous dissipation on unsteady mixed convection flow past a porous plate moving through a binary mixture of chemically reacting fluid. - Thermo Energy Power Eng, vol.2, pp.134-46. 
[10] Siddiqa S., Asghar S. and Hossain M.A. (2012): Radiation effect on mixed convection flow of viscous fluid having temperature dependent density along permeable vertical plate. - Journal of Engineering Physics and Thermo Physics, vol.85, No.2, pp.339-348. Doi: 10.1007/s10891-012-0658-1.

[11] Salomatov V.V. and Puzyrev E.M. (1971): Influence of thermal radiation on the laminar boundary layer of a nonabsorbing fluid. - Inzhenerno-Fizicheskii Zhurnal, vol.20, pp.1008-1014.

[12] Bakier A.Y (2001): Thermal radiation effect on mixed convection from vertical surfaces in saturated porous media. - Int. Comm. Heat Mass Transf., vol.28, pp.119-126.

[13] Hossain A. and Munir S. (2000): Mixed convection flow from a vertical flat plate with temperature dependent viscosity. - Int. J. Thermal Sci., vol.39, pp.173-183. DOI: 10.1016/81290-0729(00)00237-4.

[14] Gupta P.S. and Gupta A.S. (1977): Heat and mass transfer on a stretching sheet with suction or blowing. - The Canadian Journal of Chemical Engineering, vol.55, pp.744.

[15] Turkyilmazoglu M. and Pop I. (2013): Exact analytical solutions for the flow and heat transfer near the stagnation point on a stretching/shrinking sheet in a Jeffrey fluid. - International Journal of Heat and Mass Transfer, vol.57, No.1, pp.82-88.

[16] Subhas Abel M. and Mahantesh M. Nandeppanavar (2009): Heat transfer in MHD viscoelastic boundary layer flow over a stretching sheet with non-uniform heat source/sink. - Communications in Nonlinear Science and Numerical Simulation, vol.14, No.5, pp.2120-2131.

[17] Liancun Zheng, Lijuan Wang and Xinxin Zhang (2011): Analytic solutions of unsteady boundary flow and heat transfer on a permeable stretching sheet with non-uniform heat source/sink. - Communications in Nonlinear Science and Numerical Simulation, vol.16, No.2, pp.731-740.

Received: December 3, 2018

Revised: March 18, 2019 